\title{
Modelagem do Comportamento Hemodinâmico de um Paciente Virtual Cirúrgico baseado em Sistema Especialista Fuzzy.
}

\author{
Hemodynamic behavior modeling of a Virtual Surgical \\ Patient based on a Fuzzy Expert System.
}

\section{Modelado de comportamiento hemodinámico de un paciente quirúrgico virtual basado en un Sistema Esperto Fuzzy.}

Paulo V. F. Paiva

Liliane S. Machado ${ }^{2}$

Tâmela $\operatorname{Costa}^{3}$

RESUMO: A Realidade Virtual (RV) permite aos seus usuários experimentarem uma sensação de estarem imersos em cenários 3D sintéticos gerados por computação gráfica. Os chamados Ambientes Virtuais (AVs) de RV, quando aplicados à educação médica, possibilitam: treino repetitivo e o desenvolvimento de habilidades psicomotoras em procedimentos cirúrgicos sem o comprometimento de pacientes reais. Os simuladores cirúrgicos que apresentam Pacientes Virtuais (PVs), isto é, que apresentam sensibilidade fisiológica às intervenções e decisões médicas tomadas ao longo do treinamento, são mais requisitados pela possibilidade de variação de casos clínicos gerados, aumentando portanto o grau de realismo. Este trabalho tem por objetivo principal, discutir importantes aspectos sobre a modelagem do comportamento hemodinâmico de um PV cirúrgico, especificamente das variáveis de Pressão Arterial Sistólica (PAS) e Diastólica (PAD) em momentos distintos da indução anestésica. Como resultado tem-se a proposição de um modelo de PV e de uma arquitetura de integração deste a sistemas de simulação baseados em RV.

Palavras-chave: Simulação de Paciente, Simulação por Computador, Lógica Fuzzy, Anestesiologia.

\footnotetext{
ABSTRACT: The Virtual Reality (VR) allows its users to experience a sense of being immersed in synthetic 3D scenarios generated by computer graphics. The so-called Virtual Environments 1 Laboratório de Tecnologias para o Ensino Virtual e Estatística (LabTEVE). Universidade Federal da Paraíba. Campus I. Jardim Cidade Universitária. João Pessoa - PB. E-mail: paulo.fariaspaiva@gmail.com

2 Docente do Departamento de Informática e pesquisadora junto ao Laboratório de Tecnologias para o Ensino Virtual e Estatística (LabTEVE). Universidade Federal da Paraíba. Campus I. Jardim Cidade Universitária. João Pessoa - PB. E-mail: liliane@di.ufpb.br

3 Laboratório de Tecnologias para o Ensino Virtual e Estatística (LabTEVE). Universidade Federal da Paraíba. Campus I. Jardim Cidade Universitária. João Pessoa - PB. E-mail: tamela_costa@hotmail.com
} 
(VEs) based on RV can be applied to medical education, enabling: repetitive training and the development of psychomotor skills in surgical procedures without compromising real patients. Surgical simulators that feature Dynamic Virtual Patients (VPs), that is, reacts physiologically to interventions and medical decisions made during the training. These systems present more realism while it offers the possibility of varying clinical cases. This work has as main objective to discuss important issues of modeling the hemodynamic performance of a VP, specifically to simulate blood pressure values (both sistolic and diastolic variables). The model of a VP is presented as result as well as is presented an architecture for its integration to simulators based on VR.

Key-words: Patient Simulation, Computer Simulation, Fuzzy Logic, Anesthesiology.

RESUMEN: La Realidad Virtual (RV) permite a sus usuarios experimentar una sensación de estar inmersos en escenarios 3D sintéticos generados por los gráficos de computadora. Los llamados entornos virtuales (AVs) de RV cuando se aplican a la educación médica, permiten: entrenamiento repetitivo y el desarrollo de habilidades psicomotrices en procedimientos quirúrgicos sin comprometer pacientes reales. Simuladores quirúrgicos que cuentan con pacientes virtuales (PV) dinámicos, es decir, que tienen la sensibilidad fisiológica de las intervenciones y las decisiones médicas tomadas durante el entrenamiento, que son los más buscados por la posibilidad de variar los casos clínicos generados, lo que aumenta el grado de realismo. Este trabajo tiene como objetivo principal discutir aspectos importantes de modelar el comportamiento hemodinámico de un PV, específicamente las variables de la presión arterial sistólica (PAS) y diastólica (PAD). Como resultado se presenta la propuesta de un modelo PV y una arquitectura de integración de este modelo en sistemas de simulación basados en RV.

Palavras clave: Simulación de Paciente, Simulación por Computador, Lógica Fuzzy, Anestesiología.

\section{INTRODUÇÃO}

A tecnologia da Realidade Virtual (RV) é um ramo inter e multidisciplinar do conhecimento humano e que permite a simulação de eventos diversos em ambientes gráficos tridimensionais, sendo estes denominados Ambientes Virtuais (AVs) ${ }^{1}$. Algumas das principais características dos AVs são: respostas às ações do usuário em tempo real (interatividade); aguçamento da cognição dos usuários fazendo-os sentirem-se imersos em uma realidade alternativa (princípio de imersão); uso de dispositivos eletrônicos para interação homem-máquina não convencionais (ex: luvas de dados, dispositivos que exploram o tato), dentre outras ${ }^{2}$. No contexto da educação em saúde, a medicina certamente é uma das áreas mais beneficiadas com o advento desta tecnologia. Os simuladores baseados em RV voltados para a medicina (ou simuladores médicos), possibilitam a redução de custo na formação de novos médicos, o uso de técnicas de ensino mais interativa, e diminuição de uso de cobaias animais e cadáveres, favorecendo os aspectos éticos envolvidos na formação de novos profissionais ${ }^{2,3}$.

A princípio, uma simulação gráfica constitui-se de um modelo matemático e físico de um ou 
vários fenômenos naturais, bem como das interações entre diferentes eventos. Neste sentido, a RV torna possível a simulação de um ou mais sistemas fisiológicos (cardiopulmonar, respiratório, linfático, etc) de pacientes virtuais (PVs $)^{4}$. As simulações de PVs são aplicadas com diferentes objetivos: monitorização cirúrgica dos sinais vitais, treino no atendimento de emergências ${ }^{5}$, teste de reações fisiológicas a novos medicamentos, realização de diagnósticos, , treino em tomada de decisão cirúrgica, realização de diagnósticos, randomização de quadros clínicos, simulação de condições médicas extremas permitindo aos estudantes ganharem experiência, parametrização do PV com base em dados de pacientes reais, dentre outras. De modo geral, os PVs apresentam a característica de responsividade, isto é, são capazes de reagirem adequadamente à diferentes estímulos (externos ou internos) à sua própria fisiologia, tais como intervenções médicas. Estes AVs também são conhecidos como simuladores de pacientes virtuais dinâmicos (PVD), ou pacientes virtuais com fisiopatologia dinâmica ou responsiva ${ }^{4}$.

Neste contexto, diferentes Modelos de Decisão (MD) ou técnicas de Inteligência Artificial (IA) ${ }^{1,3,5}$ podem ser aplicadas na modelagem destes sistemas fisiológicos ${ }^{4}$. No entanto, antes da definição do MD a ser utilizado, faz-se necessário responder algumas importantes questões: qual a natureza dos dados envolvidos (ex: sinais vitais)? como tratá-los? apresentam (ou não) aspectos de imprecisão e/ou incerteza de mensuração? quais os eventos cirúrgicos aos quais o modelo estará subordinado? qual o nível de interação (correlação) com os outros sistemas fisiológicos do paciente? Ao longo do processo de delineamento deste estudo, foi observado que para a simulação da fisiologia de um PV, grande parte das variáveis envolvidos (ex: sinais vitais), apresenta uma natureza intrinsecamente imprecisa. Deste modo, para este trabalho, optou-se pelo uso da teoria da lógica fuzzy (difusa ou nebulosa) proposta por Lotfi Zadeh ${ }^{6}$, que afirma que onde exista subjetividade na base de conhecimento, a sua representação pode ser feita pela lógica fuzzy.

Este trabalho tem por objetivo principal apresentar uma proposta de modelagem do comportamento hemodinâmico de um PV cirúrgico (sob monitoração não-invasiva) por meio de uma pesquisa de desenvolvimento metodológico. As variáveis inicialmente estudadas são: pressão arterial sistólica (PAS) e diastólica (PAD). A monitorização da pressão arterial é o método mais usual de monitorização do sistema cardiovascular, sendo que tais variáveis representam a energia potencial para perfusão tecidual, isto é, a capacidade de irrigação sanguínea dos tecidos ${ }^{7}$. O modelo proposto é baseado em um Sistema Especialista (SE) $f u z z y^{1,6}$ sendo consultados dados reais (quali e quantitativos) encontrados na literatura médica a respeito das flutuações dos parâmetros hemodinâmicos de pacientes cirúrgicos em diferentes condições de normo ou hipertensão ${ }^{7,8}$.

\section{FUNDAMENTAÇÃO TEÓRICA}

\section{Monitoramento da Pressão Arterial do Paciente Cirúrgico}

Os elementos fundamentais da monitorização cirúrgica incluem observação e vigilância, observação de dados por meio de aparelhos, análise e instituição de medidas de correção, para 
cada caso indicado. A monitorização objetiva proporcionar as melhores condições intraoperatórias e detectar anormalidades precocemente para que sejam empregadas medidas corretivas antes de acontecer lesões sérias e irreversíveis ${ }^{9}$. Os monitores são utilizados para uma maior segurança do paciente que se submete a uma cirurgia facilitando a leitura de diferentes sinais vitais, tais como: pressão arterial, frequência cardíaca, níveis de saturação de oxigênio, frequência respiratória, temperatura corporal, dentre outros ${ }^{8}$. Indicações, riscos e benefícios relacionados ao uso de monitorização eletrônica invasiva e não invasiva devem ser avaliados conforme cada paciente individualmente. Essas decisões devem ser tomadas levando-se em consideração a condição do paciente, o tipo de cirurgia e o risco de complicações inerentes a monitorização invasiva. Todavia, a propagação de dispositivos de monitorização não reduz a necessidade de parâmetros clínicos (inspeção, observação, ausculta, palpação) ${ }^{8}$.

A pressão arterial sanguínea consiste na pressão de propulsão criada pela ação de bombeamento do coração, usualmente, a pressão sanguínea arterial reflete a pressão ventricular ${ }^{10}$. A pressão sistólica consiste na maior pressão arterial medida durante um ciclo cardíaco referente a pressão na artéria após o sangue ter sido ejetado pelo ventrículo esquerdo na sístole, enquanto a pressão diastólica, é a pressão arterial mais baixa medida durante um ciclo cardíaco equivalente a pressão na artéria durante o relaxamento ventricular quando não há mais sangue sendo ejetado pelo ventrículo esquerdo $^{11}$. Segundo as Diretrizes Brasileiras de Hipertensão estipuladas pela Sociedade Brasileira de Cardiologia ${ }^{12}$, diferentes classes são utilizadas para classificação dos níveis pressóricos de um paciente (Tabela I).

Tabela I. Classes de pressão arterial segundo as Diretrizes Brasileiras de Hipertensão.

\begin{tabular}{|l|l|l|}
\hline Classificação & PSA $(\mathrm{mmHg})$ & PSD $(\mathrm{mmHg})$ \\
\hline Ótima & $<120$ & $<80$ \\
\hline Normal & $<130$ & $<85$ \\
\hline Limítrofe & $130-139$ & $85-89$ \\
\hline Hipertensão estágio 1 & $140-159$ & $90-99$ \\
\hline Hipertensão estágio 2 & $160-179$ & $100-109$ \\
\hline Hipertensão estágio 3 & $\geq 180$ & $\geq 110$ \\
\hline $\begin{array}{l}\text { Hipertensão sistólica } \\
\text { isolada }\end{array}$ & $\geq 140$ & $<90$ \\
\hline
\end{tabular}

Fonte: VI Diretrizes Brasileiras de Hipertensão Arterial. Sociedade Brasileira de Cardiologia.

A monitorização da pressão arterial, é necessária durante todos os procedimentos anestésicos e pode ser considerado como método mais usual de monitorização do sistema cardiovascular ${ }^{7}$. As técnicas disponíveis de monitorização não invasiva da pressão arterial são apropriadas para a maioria dos casos cirúrgicos e as salas de operação mais modernas estão equipadas com analisadores da pressão arterial automatizados. As indicações para monitorização invasiva da pressão arterial incluem uso intraoperatório de hipotensão induzida, avaliação contínua da pressão arterial em 
pacientes com lesão orgânica grave ou submetidos a procedimentos cirúrgicos de alto risco quando os métodos não invasivos são inadequados ${ }^{9}$.

\section{Sistemas Especialistas (SE) e a Lógica fuzzy}

Neste contexto de modelagem do conhecimento humano, estão inseridos os Sistemas Especialistas (SEs). Segundo Moraes e $\mathrm{Machado}^{1}$, a arquitetura formada pela base do conhecimento especialista (neste caso, o conhecimento médico) e por um sistema de inferência é chamada "Sistema Especialista" (SE). Os SEs são aqueles sistemas que se utilizam do conhecimento de um especialista em um determinado domínio específico, para responder importantes questões e solucionar problemas deste domínio. Nestes sistemas, podem ser usados para a modelagem do conhecimento, a lógica clássica e/ou a lógica fuzzy, sendo este codificado em regras lógicas, sendo que cada regra de um SE diz respeito a uma variável de interesse. As regras mais básicas são do tipo "SE < condição satisfeita $>$ ENTÃO < conclusão $>$ ". A partir das regras e dos fatos representados no conhecimento especialista, fatos novos (conclusões) são obtidos através de um processo de inferência ${ }^{1}$.

A conhecida lógica fuzzy (ou nebulosa) proposta por Zadeh ${ }^{6}$, permite a tradução de informações imprecisas e vagas (como dados qualitativos) em valores numéricos que em geral são descritas em linguagem natural, e convertidas em regras de fácil manipulação pelo computador, tornando os sistemas computacionais mais aptos a tratarem tais dados. Desta forma, a lógica fuzzy torna possível a modelagem da subjetividade envolvida na experiência humana em um controle computadorizado ${ }^{1,2}$. A lógica fuzzy difere dos sistemas de lógica clássica em seu mapeamento de verdadeiro e falso. Na lógica clássica, o valor verdade só pode assumir dois valores: "verdadeiro" ou "falso" (1 ou 0), enquanto que na lógica fuzzy o valor verdade de uma proposição pode assumir diferentes níveis de pertencimento no intervalo unitário [0,1]. Matematicamente, segue a definição:

Definição 1. Tomemos U como conjunto universal, onde todos os elementos são denotados por $\mathrm{x}$. Logo, um conjunto fuzzy A em $\mathrm{U}$ é dado por: $\mathrm{A}=\{\mathrm{x}, \mu \mathrm{A}(\mathrm{x})\}, \mathrm{x} \in \mathrm{U}$.

Onde $\mu \mathrm{A}(\mathrm{x})$ é chamada a função de pertinência ou gradação de pertinência de um elemento $x$ em um conjunto fuzzy A e $\mu \mathrm{A}: \mathrm{U} \rightarrow[0 ; 1]$. O valor 0 significa que o elemento $x$ pertence absolutamente à A e o valor 1 significa que $x$ absolutamente não pertence à A. Neste sentido, esta é uma interpretação análoga à lógica clássica ${ }^{1}$.

A teoria dos conjuntos fuzzy revisita vários conceitos médicos e epidemiológicos, expandindo a ocorrência de novos insights. Segundo Massad ${ }^{13}$, por muito tempo, os conceitos de doença e saúde têm sido tratados como condições opostas pela comunidade médica. No entanto, na a abordagem de raciocínio fuzzy, estas condições são antes complementares do que contraditórios. Ou seja, determinado indivíduo pode ser classificado ao mesmo tempo, como saudável e não-saudável, em diferentes níveis de intensidade ${ }^{14}$. Mamdani ${ }^{15}$ propôs uma forma de inferência para sistemas 
baseados em regras fuzzy, baseada na t-norma "mínimo" e na t-conorma "máximo" para controle adaptativo fuzzy. Atualmente, essa forma é muito usada para modelar sistemas baseados em regras, mesmo que a aplicação não seja em controle adaptativo. Tal arquitetura pode ser vista na Figura 1 foi apresentada por Santos et al. ${ }^{16}$. :

Figura 1. Arquitetura de SE fuzzy genérico.

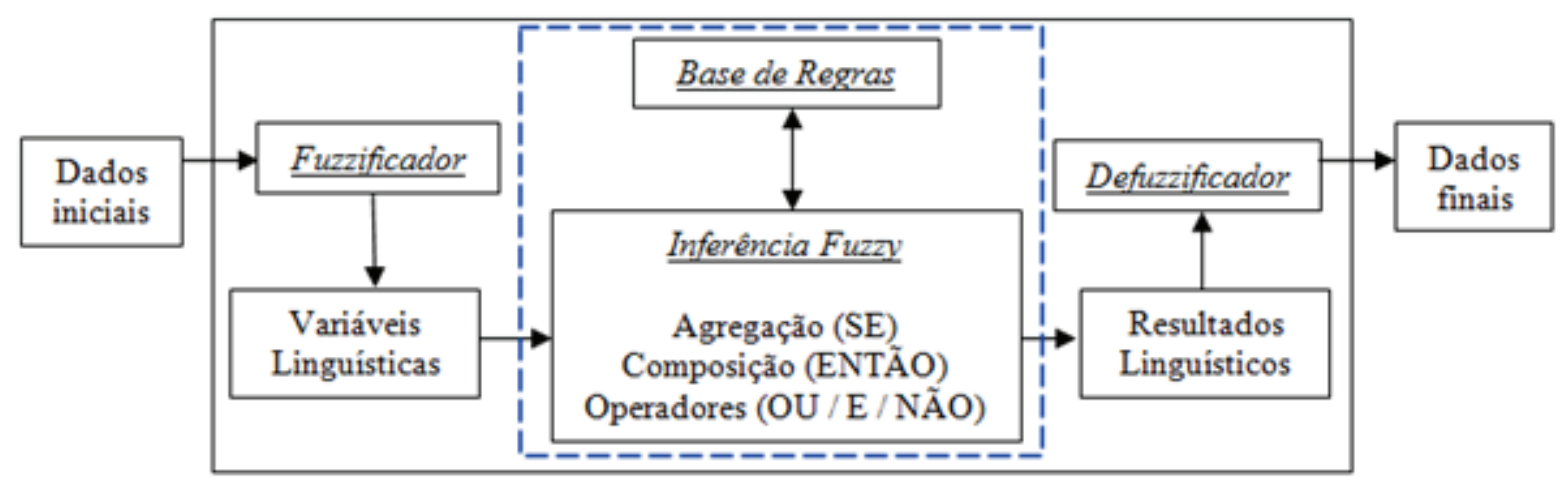

Fonte: Adaptado de Santos et al. ${ }^{16}$

a) Processo de Fuzzificação: Neste processo, os valores numéricos das variáveis de entrada são transformados em termos linguísticos. Para cada variável, são definidas também suas diferentes funções de pertinência. Consecutivamente, para cada classe de PSA, são definidos diferentes graus de pertinência para cada valor possível de PSA dentro do espaço amostral $(\Omega)^{13-15}$.

b) Base de Conhecimento Especialista (Regras): Trata-se da parte mais importante do SE, pois é nele onde as regras são implementadas. As informações sobre as regras e variáveis, podem ser obtidas de diferentes formas, tais como: baseando-se na experiência real de especialistas; com base em estudos da área e guias de referência, dentre outras ${ }^{13-15,16}$.

c) Interface de Inferência: Processamento dos dados fuzzy de entrada, junto com as regras (proposições), de modo a inferir os conjuntos fuzzy de saída, aplicando o operador para implicação. Ao longo da implicação, é realizada a obtenção das conclusões (variáveis fuzzy de saída) ${ }^{13-15}$.

d) Processo de Defuzzificação: Transforma os conjuntos fuzzy de saída, inferidos anteriormente em dados precisos novamente. Efetua um escalonamento, de modo a compatibilizar os valores normalizados vindos do passo anterior, com os valores dos universos de discurso reais das variáveis $^{13-15}$.

\section{METODOLOGIA}

A pesquisa englobou quatro etapas e iniciou-se com um levantamento bibliográfico acerca de estudos que discutiam o comportamento fisiológico de diferentes quadros clínicos em procedimentos cirúrgicos, bem como de MDs utilizados em sistemas computacionais voltados 
à simulação de tais procedimentos. Assim, o passo inicial consistiu em entender as necessidades encontradas no desenvolvimento dessas metodologias, aprofundando pesquisas do domínio através do levantamento de estudos similares, e como os MDs estão sendo utilizados nos simuladores de RV, para simulação fisiológica ${ }^{1,2}$. A partir dos resultados encontrados na pesquisa bibliográfica, foram delimitadas um escopo de estudo definindo as variáveis a serem utilizadas no modelo de um PV. Tal modelo foi utilizado, em uma terceira etapa, para especificar casos problema que mostrassem a relevância da utilização do modelo de PV. Como o estudo não está analisando empiricamente nenhum fenômeno, este texto se apresenta como pesquisa de desenvolvimento metodológico ${ }^{3}$ e não como estudo exploratório-descritivo. Após a fase inicial de delineamento, foi utilizado o software de modelagem de sistemas fuzzy denominado inFuzzy $y^{17}$ para modelagem do SE (variáveis) e algumas regras iniciais.

O levantamento dos trabalhos foi realizado com o auxílio do portal de busca de periódicos da CAPES2 e da ferramenta de busca Google Acadêmico, com buscas feitas em várias bases de publicações científicas, tais como: IEEE Xplore, Elsevier, PubMed, SpringerLink, Scielo, ACM Digital Library, CiteSeerX, dentre outras. As palavras-chave utilizadas nas buscas foram: Simulação Cirúrgica, Paciente Virtual, Simulação Hemodinâmica, Variação Cardiovascular, Monitorização Cirúrgica, Hipertensão, Realidade Virtual. Os mesmos termos em inglês foram utilizados. Buscas em diferentes momentos foram efetuadas com auxílio do recurso do portal de periódicos da CAPES, por diferentes tipos de publicações científicas (capítulos de livros, anais de congressos, artigos, dentre outros) nos últimos 5 anos (2010 - 2015). Ao todo foram encontrados 436 títulos em um primeiro momento (Março de 2015) e 769 títulos em segundo momento (Abril de 2013), dentre os quais selecionamos 25 trabalhos de referência.

Como critérios de escolha dos trabalhos, foram incluídos os estudos que envolvessem o uso da RV como ferramenta de suporte à educação e treinamento em saúde e/ou que utilizassem de MDs em sua modelagem bem como estudos da área médica. Procuramos analisar importantes requisitos dos PVs nos AVs e que relacionam-se entre si, tais como: a) objetivos da simulação; b) detalhamento fisiológico do PV; c) MD utilizados; d) variáveis envolvidas. Com base nos passos elencados previamente, pretende-se apresentar exemplos de regras de decisão do modelo onde as variáveis envolvidas assumam diferentes condições, simulando portanto, diferentes quadros clínicos.

\section{RESULTADOS}

\section{Estado da Arte: Pacientes Virtuais}

Segundo Dev et al. ${ }^{18}$, tais modelos de fisiologias virtuais podem apresentar um comportamento "estático" ou dinâmico. No primeiro caso, modelos mais simples simulam apenas um quadro clínico em particular (estado do paciente). No segundo caso, a evolução do estado do paciente é acompanhada no tempo, tanto com ou sem intervenções do estudante. No entanto, de modo 
geral, estes modelos apresentam a característica de responsividade, isto é, são capazes de reagirem adequadamente aos diferentes estímulos externos ou internos à própria fisiologia do PV. Os estímulos externos compreendem as intervenções médicas (clínicas, cirúrgicas ou farmacológicas) e/ou eventos inesperados que possam vir a interferir no estado do paciente, e os internos referemse às reações fisiológicas (adversas ou não) do modelo ${ }^{18}$. Neste tópico, serão apresentados alguns simuladores de pacientes virtuais, e que se assemelham à proposta deste trabalho. O CliniSpace ${ }^{19}$ é um simulador de um ambiente médico hospitalar e simula pacientes virtuais com fisiopatologia dinâmica, isto é, que são capazes de responderem a uma gama de medicamentos e procedimentos. O Virtual ED Patient ${ }^{18}$ é um modelo de PV simulado para cenários de medicina emergencial. Para a modelagem deste PV é utilizado como a lógica clássica, sendo monitorados quatro sinais vitais (pressão sanguínea, frequência cardíaca e respiratória e oxigenação) para representação do seu estado. Os sistemas acima citados são alimentados com base em dados quantitativos provindos da literatura médica ${ }^{18,19}$. Os simuladores $U V I M O^{16}$ e o $T O U C H^{20}$, por sua vez, simulam apenas um caso. No primeiro caso, é simulado um paciente diabético sofrendo um ataque cardíaco do miocárdio, com base em um SE fuzzy. No segundo caso, é modelado um PV com traumatismo craniano proveniente, sendo utilizada a lógica clássica como MD. Os simuladores 3 DiTeams ${ }^{21}$ e o Simulador de Cirurgias Ortopédicas ${ }^{22}$ destacam-se no âmbito dos simuladores colaborativos (que permitem o treino à distância ${ }^{23,24}$. No primeiro caso, é simulada uma sala de operações militares e possui um módulo de simulação fisiológica, com foco principal no treino de habilidades de interação em equipe. No segundo caso, é utilizada uma Árvore de Decisão ${ }^{5}$ como MD para a lógica computacional do jogo generalizando as estratégias para o gerenciamento de perda sanguínea do PV, ao longo da cirurgia ortopédica. Alguns exemplos dos sistemas aqui apresentados estão na Figura 2.

Figura 2. Exemplos de simuladores de RV com modelos de PV: CliniSpace à esquerda, UVIMO no centro e o 3 DiTeams à direita.
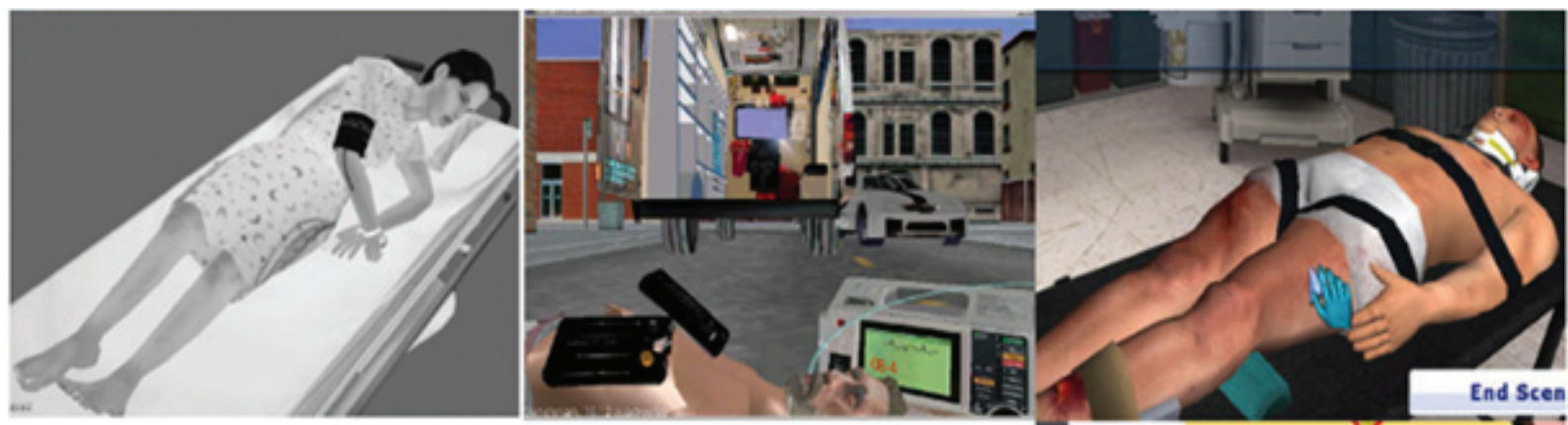
Tabela II. Quadro comparativo entre os simuladores de RV com PVs estudados.

\begin{tabular}{|c|c|c|c|c|}
\hline $\begin{array}{c}\text { Simuladores } \\
\text { de RV }\end{array}$ & Objetivo & MD & $\begin{array}{c}\text { Sinais vitais ou variáveis } \\
\text { monitoradas }\end{array}$ & $\begin{array}{c}\text { Variabilidade } \\
\text { dos casos }\end{array}$ \\
\hline CliniSpace ${ }^{18}$ & $\begin{array}{l}\text { Fisiopatologia } \\
\text { dinâmica }\end{array}$ & $\begin{array}{l}\text { Lógica } \\
\text { clássica }\end{array}$ & $\begin{array}{l}\text { Taxa de sangramento, dentre } \\
\text { outros sinais vitais }\end{array}$ & $\begin{array}{l}\text { Múltiplos } \\
\text { casos }\end{array}$ \\
\hline $\begin{array}{l}\text { Virtual ED } \\
\text { Patient }{ }^{19}\end{array}$ & $\begin{array}{l}\text { Medicina } \\
\text { emergencial }\end{array}$ & $\begin{array}{l}\text { Lógica } \\
\text { clássica }\end{array}$ & $\begin{array}{l}\text { Oxigenação sanguínea, } \\
\text { pressão arterial, frequência } \\
\text { cardíaca e respiratória, taxa } \\
\text { de sangramento. }\end{array}$ & $\begin{array}{l}\text { Múltiplos } \\
\text { casos }\end{array}$ \\
\hline UVIMO $^{16}$ & $\begin{array}{l}\text { Medicina } \\
\text { emergencial }\end{array}$ & $\begin{array}{l}\text { SE baseado } \\
\text { em lógica } \\
\text { fuzzy }\end{array}$ & $\begin{array}{l}\text { Saturação do oxigênio, nível } \\
\text { de oxigenação, temperatura, } \\
\text { PAS, PAD }\end{array}$ & $\begin{array}{l}\text { Diabético } \\
\text { sofrendo } \\
\text { infarto do } \\
\text { miocárdio }\end{array}$ \\
\hline 3DiTeams ${ }^{22}$ & $\begin{array}{l}\text { Treino de } \\
\text { equipes em } \\
\text { operações } \\
\text { militares }\end{array}$ & $\begin{array}{l}\text { Motor } \\
\text { fisiológico }\end{array}$ & $\begin{array}{l}\text { Temperatura, oxigenação } \\
\text { sanguínea, pressão arterial, } \\
\text { frequência cardíaca e } \\
\text { respiratória, taxa de } \\
\text { sangramento, pressão de } \\
\text { pulso. }\end{array}$ & $\begin{array}{l}\text { Múltiplos } \\
\text { casos }\end{array}$ \\
\hline $\begin{array}{l}\text { Cirurgias } \\
\text { Ortopédicas }{ }^{21}\end{array}$ & $\begin{array}{l}\text { Treino } \\
\text { psicomotor } \\
\text { em cirurgias } \\
\text { ortopédicas }\end{array}$ & $\begin{array}{l}\text { Árvore de } \\
\text { Decisão }\end{array}$ & $\begin{array}{l}\text { Volume sanguíneo } \\
\text { (volemia), frequência } \\
\text { cardíaca, taxa de } \\
\text { sangramento, tempo do } \\
\text { procedimento }\end{array}$ & $\begin{array}{l}\text { Diferentes } \\
\text { classes } \\
\text { de perda } \\
\text { sanguínea }\end{array}$ \\
\hline $\mathrm{TOUCH}^{20}$ & $\begin{array}{l}\text { Medicina } \\
\text { emergencial }\end{array}$ & $\begin{array}{l}\text { Lógica } \\
\text { clássica }\end{array}$ & $\begin{array}{l}\text { Tempo decorrido, pressão } \\
\text { arterial }\end{array}$ & $\begin{array}{l}\text { Traumatismo } \\
\text { Craniano }\end{array}$ \\
\hline
\end{tabular}

Como observado, dentre os simuladores estudados, possuem diferentes objetivos (Treino de equipes, medicina emergencial, e fisiopatologia dinâmica). Foi verificado que todos os simuladores se utilizam da lógica clássica como MD, sendo que o simulador de cirurgias ortopédicas se utiliza de tal modelo na codificação de uma árvore de decisão. O UVIMO é o único sistema que se utiliza da lógica fuzzy.

Dentre as variáveis monitoradas estão: saturação do oxigênio, frequência cardíaca e respiratória, taxa de sangramento, tempo do procedimento, pressões arteriais sistólica (PAS) e diastólica (PAD), volemia e saturação de oxigenação. Quanto às possibilidades de variação dos casos simulados, destacamos os simuladores CliniSpace, Virtual ED Patient e 3DiTeams, que permitem a simulação de múltiplos casos, sendo que os demais apenas simulam um único caso clínico.

\section{Proposta de um Modelo de PV com base em Lógica Fuzzy}


A elaboração do modelo dividiu-se em quatro etapas a considerar: 1- levantamento de requisitos; 2- definição das variáveis fuzzy; 3- criação do sistema de inferência (base de regras); 4- Proposta de incorporação do PV em um simulador de RV.

\section{Levantamento de requisitos}

Segundo as boas práticas de desenvolvimento de simuladores médicos e guias de referência em $\mathrm{RV}$, é recomendado a definição de todos os requisitos previamente à etapa de coleta e modelagem do conhecimento especialista médico em questão $0^{1,2}$. Para a fase de planejamento e modelagem de uma fisiologia virtual, torna-se importante a presença de uma equipe multidisciplinar, com profissionais da área médica, de computação, matemática e/ou estatística, dentre outros. Deste modo, os conhecimentos especialistas podem ser incorporados à simulação de forma mais coerente com a realidade dos fenômenos observados ${ }^{16}$.

A princípio, faz-se necessário a definição do escopo de simulação, uma vez que a representação fisiológica completamente fidedigna de um paciente cirúrgico, torna-se impraticável. Isto ocorre, uma vez que a predição de todos os possíveis estados que um (ou mais) sistema(s) fisiológico(s) $\operatorname{pode}(\mathrm{m})$ vir a assumir são praticamente infinitos, bem como o relacionamento entre outro(s) sistema(s) ${ }^{19}$. Além disto, diferentes níveis de detalhamento fisiológico são possíveis (orgânico, celular, bioquímico) e além dos eventos puramente fisiológicos (internos ao PV), existem também aqueles de natureza externa, provenientes do meio, como por exemplo, as intervenções cirúrgicas que dependem das decisões humanas.

Como observado, para modelagem de um PV, todos possíveis eventos (externos ou internos) de simulação almejados, devem ser preestabelecidos. Neste sentido, o escopo definido para este trabalho foi a simulação inicial do comportamento de flutuação de duas importantes variáveis hemodinâmicas (PAS e PAD) ao longo da indução anestésica. Os dados obtidos se basearam em estudos reais da área médica ${ }^{7,25}$. Como variável alternativa do estudo, também será analisada a volemia (volume sanguíneo) em casos extremos de hemorragias ou perda de plasma sanguíneo (choque hipo/hipervolêmico), por exemplo.

\section{Definição das Variáveis do Modelo}

A criação de um SE baseado em lógica fuzzy parte inicialmente da modelagem dos números fuzzy (ou funções de pertinência) para cada variável envolvida no estudo ${ }^{16}$. Para tal, faz-se necessário verificar quais termos linguísticos serão utilizados e quais números fuzzy são mais apropriados para defini-los numericamente. Os números fuzzy exercem um papel importante na modelagem de sistemas fuzzy, pelo fato de permitirem a quantificação de predicados qualitativos (ou termos linguísticos) e processá-los de forma matemática no processo de fuzzificação descrito anteriormente. A Figura 3 exibe as variáveis fuzzy de entrada do paciente virtual PAS e PAD, respectivamente. Os seguintes termos linguísticos foram definidos para cada função de pertinência: "muito baixa", “baixa”, "ótima”, “normal”, "limítrofe", “alta 1", “alta 2", "alta 3". Para cada variável de estudo, 
estas funções foram representados por três tipos de números fuzzy (rampa a esquerda, rampa a direita e trapézios). Como pode-se observar, a definição das classes foi embasada nas categorias de pressão estipuladas pelas Diretrizes Brasileiras de Hipertensão dispostas na Tabela 1, com exceção das funções "muito baixa" e "baixa", sendo seus valores de pertinência estipulados com base em outros estudos da área médica ${ }^{7,25}$.

Figura 3. Funções de pertinência definidos para as variáveis de Pressão Arterial Sistólica (PAS) e Diastólica (PAD).
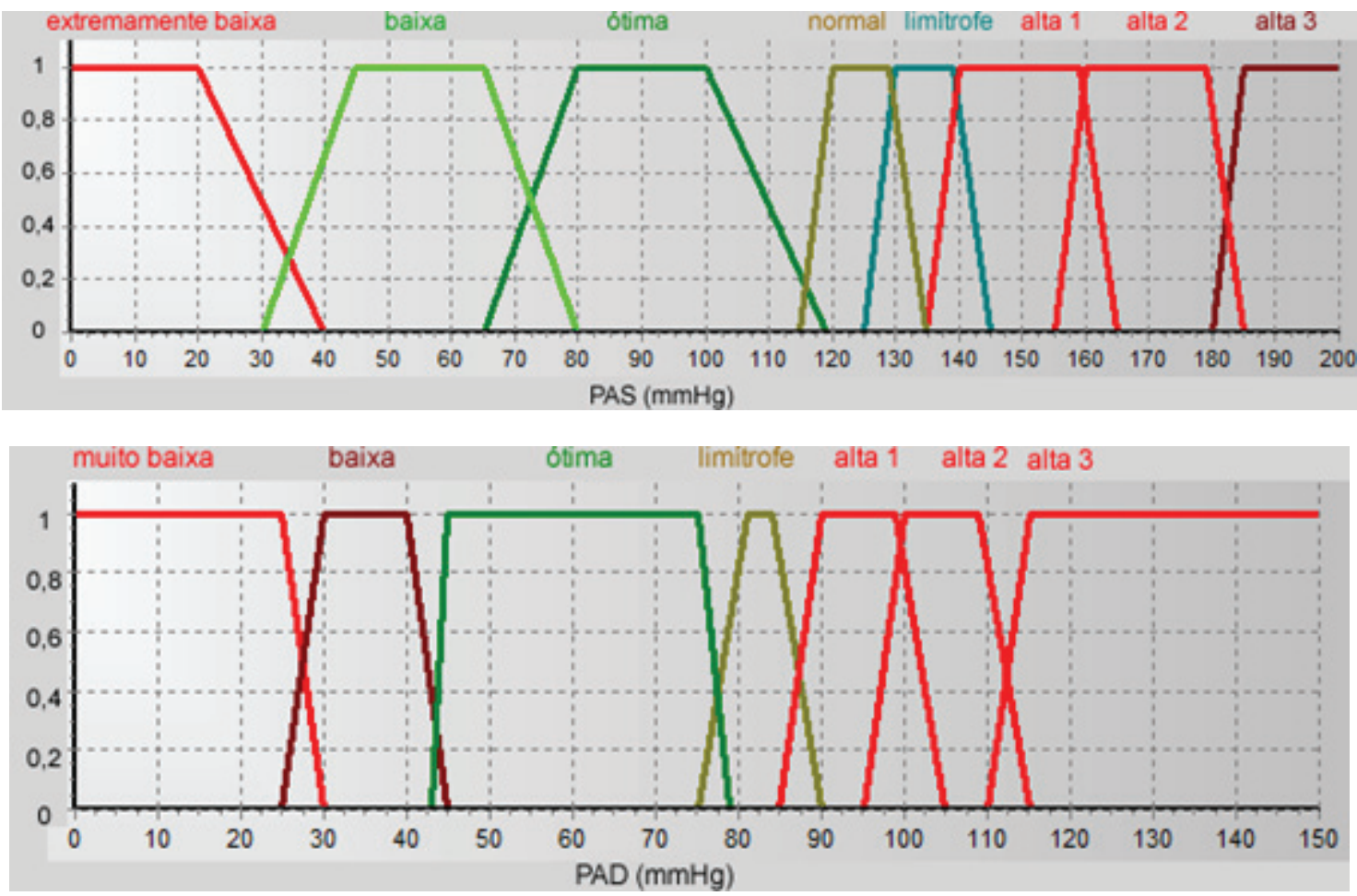

Outras importantes variáveis que se correlacionam de alguma maneira com os valores pressóricos, também podem ser mapeados em funções fuzzy, caso estas apresentem aspectos de imprecisão. Deste modo, torna-se possível a verificação de diferentes níveis de assertividade em alguns dos procedimentos observados durante a indução anestésica e monitoramento do PV. Como exemplo:

$P A S$ e PAD: São as principais variáveis de entrada do modelo, conforme apresentadas anteriormente.

infusaoDrogas: O grau de assertividade de tal procedimento é dado com base nosparâmetros de referências, tais como, a velocidade de infusão, tipo de indução (endovenosa ou inalatória) para cada caso clínico, dosagem do medicamento utilizado, etc. Poderíamos, por exemplo, definir as seguintes funções de pertinência para tal procedimento \{ incorreto, regular, correto \}.

estadoPressao: Variável de saída do SE fuzzy que define o estado atual do paciente segundo seus níveis de pressão arterial. 
estadoPaciente: Variável de saída do SE fuzzy, que define o estado atual do paciente, segundo a relação entre as demais variáveis fisiológicas definidas pelo SE. Inicialmente, foi incluída no estudo apenas a variável volemia, para fins de maior compreensão do MD proposto. Podem ser de definidas as seguintes funções de pertinência para tal variável \{muito instável, instável, estável\}.

volemia: Variável que representa os diferentes níveis de volume sanguíneo do paciente.

Outras variáveis que não possuam características de imprecisão e que pertençam ao domínio da lógica clássica, isto é, podem apenas assumir um valor no conjunto \{ "verdadeiro", "falso" \}. Geralmente estas variáveis são utilizadas para monitoramento da ocorrência de eventos diversos, como por exemplo:

choqueVolemico, hemostasia: Codificam quando há ocorrência dos eventos de choque volêmico (perda de volume e/ou plasma sanguíneo acentuada) e alcance de hemostasia (controle hemorrágico), respectivamente.

planoBailey: A ocorrência do plano anestésico de Bailey é dada quando o anestesista verifica a ocorrência de alterações fisiológicas no paciente (centralização do globo ocular, miose e arreflexia pupilar a um estímulo luminoso). A adequação a este plano, permite a continuidade da operação.

\section{Criação do Sistema de Inferência (Base de Regras)}

Como visto anteriormente, o sistema de inferência é baseado pelo encadeamento de regras, que armazenam o conhecimento médico. Por exemplo, para que o simulador de RV possa verificar se o PV já se encontra devidamente anestesiado (segundo o plano anestésico de Bailey), baseando-se nas funções de pertinência preestabelecidas, as seguintes regras apresentadas podem ser utilizadas pelo SE:

\section{SE "infusaoDrogas é correta $\mathbf{E}$ "planoBailey é verdadeiro" ENTÃO "planoAnestesico é} correto"

\section{SE “PSA é alta 3" E "PSD é limitrofe" ENTÃO "estadoPressao é alta 3"}

\section{SE "estadoPressao é alta 3" E "volemia < 20\%" ENTÃO "choqueVolêmico é verdadeiro"}

Tal como se verifica, a partir das regras, cria-se um sistema de encadeamento lógico que toma decisões de acordo com as intervenções cirúrgicas já realizadas. Um outro exemplo de situação externa ao modelo fisiológico do PV, é a verificação da decisão do estudante em relação à uma situação inesperada de hemorragia. Para tal fim, pode ser definido um vetor de dados contendo $k$ opções booleanas (com valores corretos e incorretos), para que o PV possa reagir conforme a decisão tomada a partir de uma hemorragia (representada pelo valor tratamento $[x]$ ):

tratamento $[k] \leftarrow\left\{\right.$ cristaloide, coloide, $\mathrm{CO}_{2}$, transfusaoSangue, .... $\left.k\right\}$ 
SE "choqueVolêmico é verdadeiro" $\mathbf{E}$ "tratamento $[x]$ é verdadeiro" $\mathbf{E}$ "hemostasia é verdadeiro" ENTÃO "choqueVolêmico é falso"

SE “choqueVolêmico é falso" E "volemia é normal” E "estadPressao é normal” ENTÃO "estadoPaciente é estável"

Uma questão importante a ser observada, é que para o MD não há uma distinção bem definida entre os eventos que ocorrem interna ou externamente ao PV, e que, portanto, influenciam no seu comportamento. Isto ocorre, uma vez que as variáveis de entrada do modelo são codificadas na mesma forma, independentemente das causas de sua origem (estímulos). Com base nas conclusões obtidas, a partir das premissas, o SE realiza o processo de defuzzificação dos termos linguísticos (ou variáveis de saída fuzzy), simulando as reações fisiológicas do paciente.

\section{Adequação de Simuladores para Uso do PV}

Para futura incorporação do modelo de PV em um simulador genérico, far-se-ão necessárias modificações na arquitetura de software, como por exemplo, a inserção de algumas funcionalidades, que serão elencadas nesta seção. Como estudo de caso, iniciamos o desenvolvimento de algumas delas no Simulador Colaborativo para Educação Cirúrgica $(\operatorname{SimCEC})^{24}$. O SimCEC permite o treinamento e avaliação de um grupo de estudantes em procedimentos básicos cirúrgicos. A arquitetura proposta é apresentada a seguir (Figura 4):

Figura 4. Proposta de arquitetura de simulador de RV para incorporação do modelo de PV.

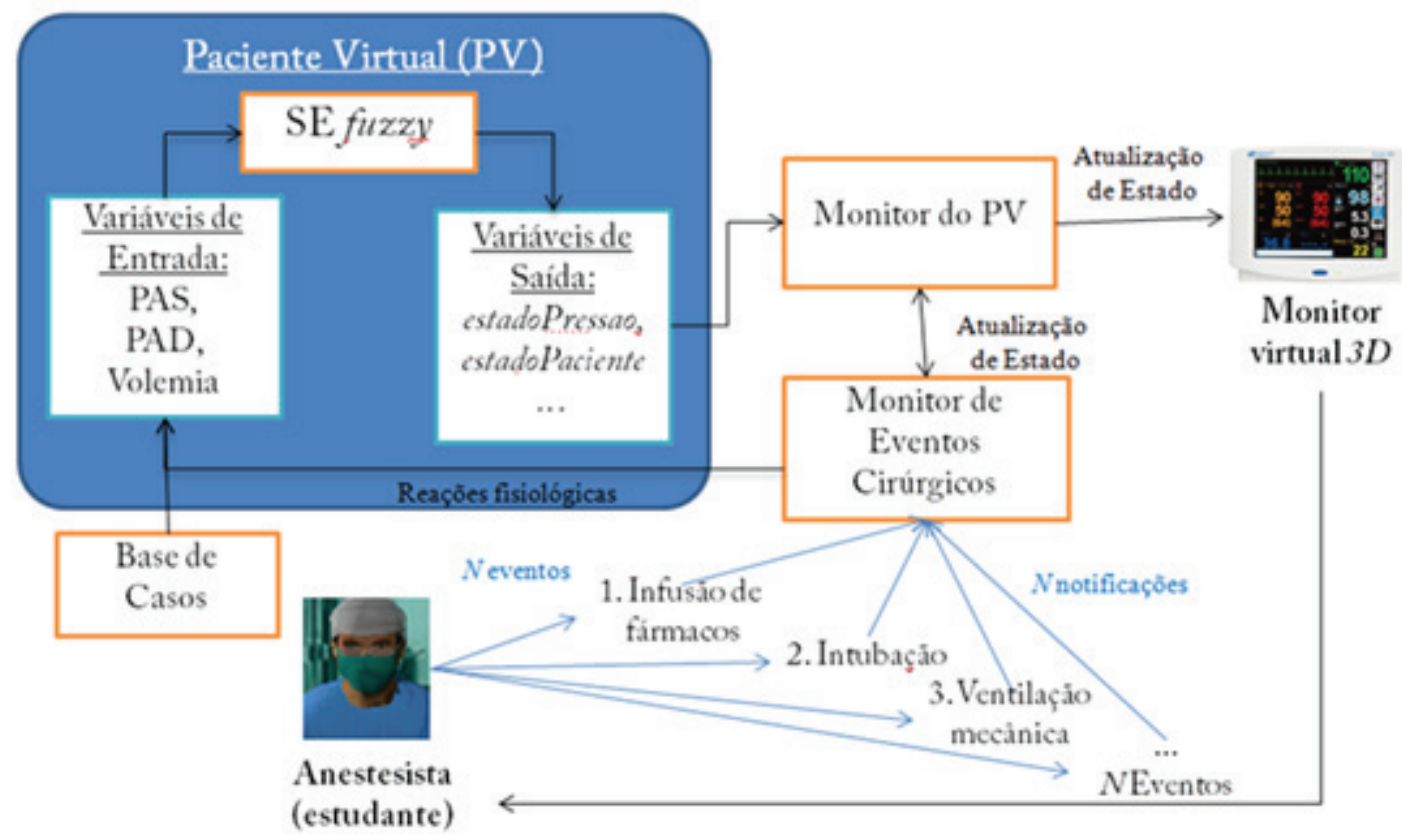

Fonte: Elaborado pelos autores (2015).

a) Monitor de Eventos Cirúrgicos: Responsável por detectar a ocorrência de todos eventos cirúrgicos previstos, tais como indução anestésica, intubação, dentre outros. Este módulo recebe 
novas notificações de cada ocorrência de intervenções cirúrgicas realizadas pelos estudantes no simulador. Ao mesmo tempo, são emitidas requisições (ou atualização de estados) ao "Módulo de Monitoração do $P V^{\prime}$ ' do estado fisiológico do $\mathrm{PV}$, para verificação das suas flutuações hemodinâmicas.

b) Módulo Monitor do PV: Realiza a monitoração de reações fisiológicas do paciente, com base na ocorrência (ou não) de novos eventos cirúrgicos (eventos externos ao PV). Ou seja, este módulo realiza a monitoração dos dados numéricos de saída do SE fuzzy do PV,

obtidos pelo processo de defuzzificação descrito anteriormente. Após, a visualização de tais dados é atualizada no objeto virtual $3 D$ que representa a tela de monitoramento anestésico do paciente, para exibir os valores de PSA/PSD obtidos.

c) Base de Casos: Este é um banco de dados, contendo valores de diferentes variáveis de inúmeros casos clínicos. Estes podem ser gerados aleatoriamente, ou serem obtidos a partir de bases de dados epidemiológicas reais.

d) PV (SE fuzzy): Trata-se do modelo fisiológico apresentado. O modelo é alimentado pela "Base de Casos" no início da simulação, onde um caso é gerado aleatoriamente ou definido pelos estudantes, que desejam atuar sobre determinado tipo de paciente. Além deste caso, o PV recebe como dados de entrada, as alterações fisiológicas previstas para cada sinal vital de acordo com as intervenções cirúrgicas que são notificadas ao "Monitor de Eventos Cirúrgicos".

\section{CONCLUSÕES}

É notória a utilização cada vez mais crescente dos simuladores de RV voltados para o treinamento de habilidades em procedimentos de saúde nas últimas décadas. No entanto, os simuladores são muito requisitados para educação, apesar de serem desenvolvidos com pouca atenção à etapa de planejamento e ao currículo médico ${ }^{1,2}$. Mesmo assim, o uso dos sistemas de RV tem se expandido e sido recomendado por vários órgãos relacionados à educação médica, como ferramenta auxiliar no processo de ensino-aprendizagem. Observando-se tais importantes questões, uma série de requisitos foram levantados na literatura médica e da computação (especialmente de RV), acerca dos padrões de referência para o desenvolvimento de um modelo fisiológico virtual. Observando-se a natureza de imprecisão de mensuração da maior parte das variáveis hemodinâmicas, propomos o uso da lógica $f u z z y^{6}$. O modelo de PV proposto é fisiologicamente dinâmico, ou seja, é sensível às intervenções realizadas pelos estudantes que aprendem ao interagir com os ambientes de RV. Inicialmente, foram incluídas no estudo as variáveis do sistema cardiovascular PAS e PAD. Tomamos como referência, as classes preconizadas pelas Diretrizes Brasileiras de Hipertensão Arterial $^{12}$.

Como principais contribuições do presente trabalho, destacam-se: 1- Levantamento do estado da arte e análise comparativa de simuladores médicos contendo PV; 2- Análise comparativa 
de Métodos de Decisão (MDs) utilizados nestes sistemas; 3- Modelagem de um SE fuzzy para simulação da fisiologia hemodinâmica de um PV; e 4-Definição de uma arquitetura de software para incorporação do PV concebido em simuladores médicos.

A vantagem de utilização do SE fuzzy neste caso se dá pelo fato de o paciente conseguir reagir hemodinamicamente às flutuações pressóricas, com base em seus valores incertos. Como se observou, a abordagem da lógica fuzzy, permite a ocorrência de insights importantes para a área médica, tais como a revisitação de conceitos anteriormente tidos como antagônicos como doença e saúde, pelo método tradicional de classificação. No entanto, os fenômenos do mundo real apresentam intrinsecamente aspectos de subjetividade e incertezas. É importante trazer aqui as limitações da proposta.

Foi realizada a modelagem das variáveis fuzzy de entrada e saída do SE, bem como a definição do sistema de inferência (base de regras), com base nos conhecimentos especialistas obtidos na literatura. Uma vez modelado o SE fuzzy, foi proposta uma arquitetura de software genérica para incorporação de tal modelo de PV em Simuladores Médicos. Deste modo, torna-se possível que este modelo seja genérico e possa ser utilizado por um ou mais simuladores, bastando para isto que os desenvolvedores dos simuladores médicos, definam uma base de dados, contendo as variáveis que se desejam simular. Esta base de dados, pode ser alimentada a partir de dados reais, ou dados provenientes da literatura médica.

\section{AGRADECIMENTOS}

Este projeto teve apoio da Capes e do CNPq através do projeto 310561/2012-4 e está relacionado ao Instituto Nacional de Ciência e Tecnologia (Medicina Assistida por Computação Científica Processo CNPq 181813/2010-6.

\section{REFERÊNCIAS BIBLIOGRÁFICAS}

1. Moraes, R.M; Machado, L.S. Simultaneous assessment of teams in collaborative virtual environments using Fuzzy Naive Bayes. 2013 Joint IFSA World Congress and NAFIPS Annual Meeting (IFSA/NAFIPS), p. 1343-1348, 2013.

2. Paiva, P.V.F.; Machado, L.S, Oliveira, J.C. A peer-to-peer Multicast Architecture for Supporting Collaborative Virtual Environments (CVEs) in Medicine. In: Proceedings of XIV Symposium on Virtual and Augmented Reality. Niterói/RJ, Brasil, 2012, p. 165-173.

3. Paiva, P.V.F.; Um Ambiente Virtual Colaborativo para Educação Cirúrgica. [dissertação] [internet] Universidade Federal da Paraíba; 2014. [acesso em 20 de jan. 2015]. Disponível em: http://tede.biblioteca.ufpb.br/handle/tede/6555.

4. Robert Kleinert; Roger Wahba; De-Hua Chang et. al. 3D Immersive Patient Simulators and 
Their Impact on Learning Success: A Thematic Review. Journal of Medical Internet Research. 2015, 8;17(4):e91. doi: 10.2196/jmir.3492.

5. Soares, R.A.S Modelo de Suporte à Decisão Aplicado ao Atendimento das Vítimas de Acidentes de Trânsito na Cidade de João Pessoa. [dissertação] [internet] Universidade Federal da Paraíba; 2012. [acesso em 29 de dez. 2014]. Disponível em: http://bdtd.biblioteca.ufpb.br/ tde_busca/arquivo.php?codArquivo=2381.

6. Zadeh, L.A. Fuzzy Sets. Information Control. 1965, 8(3), 338-353.

7. Silva Neto WV, Azevedo GS, Coelho FO, Netto EM, Ladeia AM. Evaluation of Hemodynamic Variations during Anesthetic Induction in Treated Hypertensive Patients. Rev. Bras. Anestesiol. 2008; 58(4): 330-341.

8. Smeltzer SC, Bare BG. Brunner \& Suddarth: tratado de enfermagem médico-cirúrgica. 12 ed. Rio de Janeiro: Guanabara Koogan, 2012.

9. Sabiston, D.C. Townsend, C.M. Tratado de Cirurgia. A Base Biológica da Prática Cirúrgica Moderna. Rio de Janeiro : Elsevier, 2010; 1(18):411-413.

10. Silverthorn, DU. Fisiologia Humana: uma abordagem integrada. Porto Alegre: Artmed, 2010 .

11. Costanzo, LS. Fisiologia. $5^{\text {a }}$ ed. Rio de Janeiro: Elsevier, 2014.

12. Sociedade Brasileira de Cardiologia, Sociedade Brasileira de Hipertensão. VI Diretrizes Brasileiras de Hipertensao Arterial. Arq Bras Cardiol 2010; 95(Supl. 1):1-51.

13. Massad, E. et al. Fuzzy logic in action: Applications in epidemiology and beyond. Studies in Fuzziness and Soft Computing, vol. 232, Springer; 2008.

14. Moraes, R.M; Machado, L.S. Fuzzy Continuous Evaluation in Training Systems Based on Virtual Reality. In: Proc. of 2009 IFSA World Congress, Lisboa, p. 102-107, 2009.

15. E.H. Mamdani, S. Assilian. An experiment in linguistic synthesis with a fuzzy logic controller. International Journal of Man-Machine Studies, v. 7, n. 1, 1975, p. 1-13.

16. Santos, A.D.; Gomes, R.G.S.; Moraes, R.M.; Machado, L.S.; A Fuzzy Logic Based Assessment Tool for VR Simulated Medical Environments. In: Proc. X Safety, Health and Environment World Congress, São Paulo, Brazil, 2010.

16. Viciana-abad; Reyes-lecuona, A. Patient modelling using expert systems for medical training simulations based on virtual reality. Anais $d$ 7th International Conference on Virtual 
Reality, VRIC - LAVAL VIRTUAL, 2005.

17. Posselt E.L., Frozza, R.; Molz, R.F. INFUZZY: Ferramenta para Desenvolvimento de Aplicações de Sistemas Difusos. Revista Brasileira de Computação Aplicada, Passo Fundo, Abril, 2015; 7(1): 42-52.

18. Dev P, Heinrichs WL, Youngblood P, Kung S, Cheng R, Kusumoto L, Hendrick A. Virtual patient model for multi-person virtual medical environments. AMIA Annu Symp Proc. 2007, Oct, 11:181-5.

19. Dev P, Heinrichs WL, Youngblood P. CliniSpace ${ }^{\mathrm{TM}}$ : A Multiperson 3D Online Immersive Training Environment Accessible through a Browser. Stud Health Technol Inform. 2011;163:1739.

20. Caudell TP; Summers KL; Holten J; Hakamata T; Mowafi M; Jacobs J; Lozanoff BK; Lozanoff S; Wilks D; Keep MF; Saiki S; Alverson D, Virtual patient simulator for distributed collaborative medical education. Anat Rec B New Anat. 2003, Jan; 270(1):23-9.

21. Taekman, J.M. et al. 3DiTeams - Healthcare team training in a virtual environment. The Journal of the Society for Simulation in Healthcare, 2008, 3(5). p. 112.

22. Cecil, J. et al Collaborative virtual environments for orthopedic surgery. 2013 IEEE International Conference on Automation Science and Engineering (CASE), p.133-137, 2013. doi:10.1109/CoASE.2013.6654045.

23. Paiva, P.V.F.; Machado, L.S.; Valença, A.M.G. A Virtual Environment for Training and Assessment of Surgical Teams. In: Anais do XV Symposium on Virtual and Augmented Reality 2013. Cuiabá/MT - Brazil. 2013; (15):1, 17-26. doi: 10.1109/SVR.2013.22.

24. Paiva, P.V.F.; Machado, L.S.; Batista, T.V.V. A Collaborative and Immersive VR Simulator for Education and Assessment of Surgical Teams. In: Anais do XVII Symposium on Virtual and Augmented Reality 2015. São Paulo/SP - Brazil. p. 176-185

25. S. Singh e J. E. Smith. Cardiovascular changes after the three stages of nasotracheal intubation. British Journal of Anaesthesia (BJA). 2003; 91(5): 667-671. doi: 10.1093/bja/aeg240.

Artigo apresentado em 31/05/15

Artigo aprovado em 31/03/16 Artigo publicado no sistema em 31/03/16 